\title{
Scattering and Transformation of Waves on Heavy Particles in Magnetized Plasma
}

\author{
Hrachya B. Nersisyan, Hrant H. Matevosyan \\ Institute of Radiophysics and Electronics, Ashtarak, Armenia \\ E-mail: hrachya@irphe.am \\ Received November 2, 2010; revised January 3, 2011; accepted January 5, 2011
}

\begin{abstract}
The scattering and transformation of the waves propagating in magnetized plasma on a heavy stationary charged particle located at a plane plasma-vacuum boundary is considered. The scattering (transformation) occurs due to the nonlinear coupling of the incident wave with the polarization (shielding) cloud surrounding the particle. It is shown that the problem is reduced to the determination of the nonlinear (three index) dielectric tensor of magnetized plasma. The angular distribution and the cross section for scattering (transformation) of high-frequency ordinary and extraordinary waves and low-frequency upper-hybrid, low-hybrid, and magnetosonic waves are investigated within a cold plasma (hydrodynamic) model.
\end{abstract}

Keywords: Scattering, Transformation, Plasma Modes, Magnetized Plasmas

\section{Introduction}

It is well known that in a medium with a certain fluctuation level, the propagation of electromagnetic waves can lead to radiation of waves with new frequencies and wave numbers, i.e. scattered waves and also a new type of wave: transformed waves. The investigations of electromagnetic waves scattering and transformation processes are very important for studying such problems as plasma diagnostics, wave transformation mechanisms in plasma, definition of dispersion properties of plasma wave processes etc. In addition the study of the electromagnetic wave scattering spectra (both in laser and microwave wave ranges) is an efficient method of plasma diagnostics in laboratory fusion research devices as well as in the near and outer space.

Electromagnetic wave scattering is caused by thermal fluctuations of plasma density and other plasma parameters such as current density, electric and magnetic fields, etc. Spectra of scattered waves provide information on the density and temperature distributions in the plasma. A peculiarity of electromagnetic wave scattering in plasmas is coherent scattering by collective plasma excitations-combination scattering that occurs along with Thompson incoherent scattering by individual plasma particles. Wave scattering by collective plasma fluctuations, in particular, makes it possible to find relative concentrations of charged particles and temperatures of individual plasma components. The phenomenon of electromagnetic wave combination scattering by collective plasma excitations has been considered for the first time by Akhiezer et al. (see, e.g., [1]). Subsequently a theory of electromagnetic wave scattering in plasmas has been developed [1-7]. The detailed theory of scattering and transformation of waves in magnetized plasma has been worked out in $[8]$ (see also $[9,10]$ where useful reviews of the electromagnetic wave scattering problem have been presented). The theory has been further developed and extended in the papers [11-15] (see also references therein), in particular, in the case of strongly magnetized turbulent plasma [14]. The scattering and transformation of high-frequency waves in dusty plasmas due to electron density inhomogeneities have been investigated in [15]. The scattering and transformation cross sections for two cases (the electrons in the shielding clouds around the charged dust particles and induced electron density fluctuations discreteness) have been calculated and it has been shown that both can be enhanced with respect to scattering from thermal fluctuations.

Another important mechanism for the wave scattering and transformation could be provided by the nonlinear interaction of the incident wave with the non-thermal density fluctuations (wakefield excitations) generated by the charged test particles moving in plasma. In particular, if such particle is at rest and heavy and does not oscillate 
in the electromagnetic field of the incident wave the scattering occurs due to the nonlinear oscillation of the polarization (shielding) cloud surrounding the particle. It is clear that the cross section of this process essentially differs from the standard Thomson cross section when the wavelength of the incident wave is comparable or smaller the size of the polarization cloud which is typically given by the Debye screening length. Moreover, in a nonlinear regime the polarization cloud surrounding the particle may be affected by an external strong magnetic field which introduces a strong anisotropy in the screening properties of the plasma and as a result in angular distribution of the scattered (transformed) waves.

In this paper we investigate this process in detail assuming that the heavy test particle is located at the plane boundary of plasma-vacuum interface. The plasma is assumed to be strongly magnetized so that the cyclotron frequency of the electrons is comparable or even larger than the plasma frequency. The case in which the incident wave propagates across the external magnetic field is considered. Within cold plasma model general expressions are obtained for the angular distribution and the cross section for the scattered and transformed waves. The explicit calculations are done for specific high-frequency ordinary and extraordinary waves as well as for low-frequency upper-hybrid, lower-hybrid, and magnetosonic waves.

\section{Theoretical Model}

The main problem in calculating the quantitative characteristics of electromagnetic wave scattering and transformation in plasmas is to find the current produced by the nonlinear interaction of the incident wave with the fluctuations of plasma parameters caused by the test particle. This current determines the scattered and transformed wave fields.

We consider an incident wave $\boldsymbol{E}^{(0)}=\boldsymbol{\varepsilon}_{0} e^{i \mathbf{k}_{0} \cdot \mathbf{r}-i \omega_{0} t}+$ c.c. (where $\varepsilon_{0}$ is the complex amplitude) which propagates in magnetized homogeneous plasma and a heavy particle with charge $\mathrm{Ze}$ ( $-e$ is the charge of an electron) at rest. The amplitude of the magnetic field of the incident wave is determined by the Maxwell's equation and has the form $\boldsymbol{B}_{0}=\left(c / \omega_{0}\right)\left[\boldsymbol{k}_{0} \times \boldsymbol{\varepsilon}_{0}\right]$. In the linear approximation the incident wave and the electric field $\delta \boldsymbol{E}(\boldsymbol{r})$ produced by the test particle are independent, and the Fourier transformed total electric field in plasma is

$$
\begin{aligned}
& \boldsymbol{E}^{(1)}(\boldsymbol{k}, \omega)=\boldsymbol{\varepsilon}_{0} \delta\left(\boldsymbol{k}-\boldsymbol{k}_{0}\right) \delta\left(\omega-\omega_{0}\right) \\
& +\boldsymbol{\varepsilon}_{0}^{*} \delta\left(\boldsymbol{k}+\boldsymbol{k}_{0}\right) \delta\left(\omega+\omega_{0}\right)+\delta \boldsymbol{E}(\boldsymbol{k}) \delta(\omega) .
\end{aligned}
$$

The amplitude and the frequency of the incident wave, for given values of the wave vector, are determined from the equations $M_{i j}\left(\boldsymbol{k}_{0}, \omega_{0}\right) \varepsilon_{0 j}=0$ and
$\operatorname{det}\left|M_{i j}\left(\boldsymbol{k}_{0}, w_{0}\right)\right|=0$, respectively, where

$$
M_{i j}(\boldsymbol{k}, \omega)=\delta_{i j}-\frac{\omega^{2}}{k^{2} c^{2}} \varepsilon_{i j}(\boldsymbol{k}, \omega)-\frac{k_{i} k_{j}}{k^{2}}
$$

and $\delta_{i j}$ are the Maxwellian and unit tensors, respectively, and $\varepsilon_{i j}(\boldsymbol{k}, \omega)$ is the dielectric tensor of the magnetized plasma.

The electric field of the stationary heavy particle is expressed by the equations $[16,17]$

$$
\delta \boldsymbol{E}(\boldsymbol{k})=-\frac{4 \pi i Z e \boldsymbol{k}}{(2 \pi)^{3} k^{2} \boldsymbol{\varepsilon}(\boldsymbol{k}, 0)} .
$$

Here $\boldsymbol{\varepsilon}(\boldsymbol{k}, \omega)=k_{i} k_{j} \boldsymbol{\varepsilon}_{i j}(\boldsymbol{k}, \omega) / k^{2}$ is the longitudinal dielectric function of the plasma. We assume that the particle is heavy and does not oscillate in the field of the incident wave. Thus the scattering originates from oscillations of the polarization cloud surrounding the particle.

To find the electromagnetic field of the scattered (transformed) wave, we consider the second order approximation in Maxwell's equations. As a result, for the electric field $E_{j}^{(2)}(\boldsymbol{k}, \omega)$ in the second order approximation we obtain the equation

$$
M_{i j}(\boldsymbol{k}, \omega) E_{j}^{(2)}(\boldsymbol{k}, \omega)=\frac{4 \pi i \omega}{k^{2} c^{2}} J_{i}(\boldsymbol{k}, \omega),
$$

where

$$
\begin{aligned}
J_{i}(\boldsymbol{k}, \omega) & =\frac{\omega}{4 \pi i} \int d \boldsymbol{k}^{\prime} \int d \omega^{\prime} \boldsymbol{\varepsilon}_{i j k}\left(\boldsymbol{k}, \omega ; \boldsymbol{k}^{\prime}, \omega^{\prime}\right) \\
& \times E_{j}^{(1)}\left(\boldsymbol{k}^{\prime \prime}, \omega^{\prime \prime}\right) E_{k}^{(1)}\left(\boldsymbol{k}^{\prime}, \omega^{\prime}\right)
\end{aligned}
$$

is the nonlinear current, associated with the nonlinear, three-index, dielectric tensor $\varepsilon_{i j k}\left(\boldsymbol{k}, \omega ; \boldsymbol{k}^{\prime}, \omega^{\prime}\right)$ of the magnetized plasma, $\boldsymbol{k}^{\prime \prime}=\boldsymbol{k}-\boldsymbol{k}^{\prime}$, and $\omega^{\prime \prime}=\omega-\omega^{\prime}$.

The scattered waves originate from nonlinear coupling of the incident wave with the electric field of the test particle. The scattering current corresponding to such coupling is easily obtained from (1) and (5) if in the expression obtained for the current we neglect the terms proportional to $\varepsilon_{0 j} \varepsilon_{0 k}$ and $\delta E_{j}\left(\boldsymbol{k}^{\prime \prime}\right) \delta E_{k}\left(\boldsymbol{k}^{\prime}\right)$, which determine the second harmonic generation and the second order electric field of the test particle, respectively. The total scattering current is thus determined by the equation

$$
\begin{gathered}
J_{i}^{(s)}(\boldsymbol{k}, \omega)=\frac{\omega}{4 \pi i}\left[S_{i j l}\left(\boldsymbol{k}, \omega_{0} ; \boldsymbol{k}_{0}, \omega_{0}\right)\right. \\
\times \delta E_{j}\left(\boldsymbol{k}-\boldsymbol{k}_{0}\right) \mathcal{E}_{0 l} \delta\left(\omega-\omega_{0}\right) \\
\left.+S_{i j l}\left(\boldsymbol{k},-\omega_{0} ;-\boldsymbol{k}_{0},-\omega_{0}\right) \delta E_{j}\left(\boldsymbol{k}+\boldsymbol{k}_{0}\right) \mathcal{E}_{0 l}^{*} \delta\left(\omega+\omega_{0}\right)\right],
\end{gathered}
$$

where the tensor $S_{i j l}$ characterizes the nonlinear properties of the medium [18]:

$$
S_{i j l}\left(\boldsymbol{k}, \omega ; \boldsymbol{k}^{\prime}, \omega^{\prime}\right)=\boldsymbol{\varepsilon}_{i j l}\left(\boldsymbol{k}, \omega ; \boldsymbol{k}^{\prime}, \omega^{\prime}\right)+\boldsymbol{\varepsilon}_{i l j}\left(\boldsymbol{k}, \omega ; \boldsymbol{k}^{\prime \prime}, \omega^{\prime \prime}\right) .
$$

The electric field $E_{i}^{\prime}(\boldsymbol{k}, \omega)$ of the scattered wave is 
obtained from Maxwell's Equation (4), in which $\boldsymbol{J}(\boldsymbol{k}, \omega)$ is replaced by the scattering current $\boldsymbol{J}^{(s)}(\boldsymbol{k}, \omega)$. Thus

$$
E_{i}^{\prime}(\boldsymbol{k}, \omega)=\frac{4 \pi i \omega}{k^{2} c^{2}} T_{i j}(\boldsymbol{k}, \omega) J_{j}^{(s)}(\boldsymbol{k}, \omega) .
$$

Here $T_{l i}(\boldsymbol{k}, \omega)$ is the tensor inverse to the Maxwellian tensor, $T_{l i}(\boldsymbol{k}, \omega) M_{i j}(\boldsymbol{k}, \omega)=\delta_{l j}$.

Since the intensity $W_{s}$ of the scattered radiation is equal to (with the minus sign) the work performed by the source of the scattered radiation per unit time, neglecting damping of the scattered wave, we obtain

$$
\begin{aligned}
W_{s}=-\frac{2 i Z^{2} e^{2} \omega_{0}^{3}\left|\boldsymbol{\varepsilon}_{0}\right|^{2}}{(2 \pi)^{2} c^{2}} \int \frac{d \boldsymbol{k}}{k^{2}} \frac{A_{i}(\boldsymbol{k}) A_{j}^{*}(\boldsymbol{k})}{\left(\boldsymbol{k}-\boldsymbol{k}_{0}\right)^{4}\left|\boldsymbol{\varepsilon}\left(\boldsymbol{k}-\boldsymbol{k}_{0}, 0\right)\right|^{2}} \\
\times \operatorname{Im}\left[T_{j i}\left(\boldsymbol{k}, \omega_{0}\right)-T_{i j}^{*}\left(\boldsymbol{k}, \omega_{0}\right)\right],
\end{aligned}
$$

where $A_{j}(\boldsymbol{k})=S_{i s l}\left(\boldsymbol{k}, \omega_{0} ; \boldsymbol{k}_{0}, \omega_{0}\right)\left(k_{s}-k_{0 s}\right) \mathrm{e}_{l}, \boldsymbol{e}=\boldsymbol{\varepsilon}_{0} /\left|\boldsymbol{\varepsilon}_{0}\right|$ being the complex unit vector along the direction of the polarization of the incident wave. As one would expect, it is seen from (9) that the scattering (transformation) on a stationary charge occurs with no frequency change $\left(\omega^{\prime}=\omega_{0}\right)$.

The total scattering cross section $\sigma$ is the ratio of the intensity $W_{s}$ of the scattered radiation to the energy flux $S=\left(c\left|\varepsilon_{0}\right|^{2} / 2 \pi\right) S_{0}$ in the incident wave, where

$$
\boldsymbol{S}_{0}=\frac{\boldsymbol{v}_{g}}{2 \omega_{0} c} \frac{\partial}{\partial \omega_{0}}\left[\omega_{0}^{2} e_{i} e_{j}^{*} \varepsilon_{i j}^{(\mathrm{H})}\left(\boldsymbol{k}_{0}, \omega_{0}\right)\right] \text {. }
$$

Here $\varepsilon_{i j}^{(\mathrm{H})}(\boldsymbol{k}, \omega)$ is the Hermitian part of the dielectric tensor and $\boldsymbol{v}_{g}=\partial \omega_{0} / \partial \boldsymbol{k}_{0}$ is the group velocity of the wave.

Assuming the group velocities of the incident and scattered waves to be considerably larger than the electron thermal velocity, we use cold plasma approximation. Within this model we write the expression for the linear dielectric tensor in the form $[17,18]$

$$
\varepsilon_{i j}(\omega)=\varepsilon_{1}(\omega) \delta_{i j}-\varepsilon_{2}(\omega) b_{i} b_{j}+i \varepsilon_{3}(\omega) e_{i j l} b_{l},
$$

where $\boldsymbol{b}$ is the unit vector in the direction of the external magnetic field, $e_{i j l}$ is a fully antisymmetric unit tensor, and

$$
\begin{gathered}
\varepsilon_{1}(\omega)=1+\sum_{a} \frac{\omega_{p a}}{\omega} g_{a}(\omega), \quad \varepsilon_{2}(\omega)=\sum_{a} \frac{\omega_{p a}}{\omega} h_{a}(\omega), \\
\varepsilon_{3}(\omega)=\sum_{a} \frac{\omega_{p a}}{\omega} l_{a}(\omega), \\
g_{a}(\omega)=\frac{\omega_{p a}(\omega+i v)}{\omega_{c a}^{2}-(\omega+i v)^{2}}, \quad l_{a}(\omega)=\frac{\omega_{c a} \omega_{p a}}{\omega_{c a}^{2}-(\omega+i v)^{2}}, \\
h_{a}(\omega)=\frac{\omega_{c a}^{2} \omega_{p a}}{(\omega+i v)\left[\omega_{c a}^{2}-(\omega+i v)^{2}\right]} .
\end{gathered}
$$

The summation in (12) and (13) is carried out over all plasma species $a, \omega_{p a}$ and $\omega_{c a}=e_{a} B_{0} / m_{a} c$ are the plasma and cyclotron frequencies of particles of the kind $a$, and $v$ is the effective frequency of electron-ion collisions.

For the vector $\boldsymbol{S}_{0}$ we obtain from (10) and (11)

$$
\begin{gathered}
\boldsymbol{S}_{0}=\frac{\boldsymbol{v}_{g}}{2 \omega_{0} c} \frac{\partial}{\partial \omega_{0}} \operatorname{Re}\left\{\omega _ { 0 } ^ { 2 } \left[\varepsilon_{1}\left(\omega_{0}\right)-|\boldsymbol{b} \cdot \boldsymbol{e}|^{2} \varepsilon_{2}\left(\omega_{0}\right)\right.\right. \\
\left.\left.+i \varepsilon_{3}\left(\omega_{0}\right)\left(\boldsymbol{e} \cdot\left[\boldsymbol{e}^{*} \times \boldsymbol{b}\right]\right)\right]\right\} .
\end{gathered}
$$

An expression for the tensor $S_{i p l}$ in the cold plasma approximation and in the absence of particle collisions was obtained in [18]. With allowance for the collisions, the expression for $S_{i p l}$ takes the form

$$
\begin{gathered}
S_{i p l}\left(\boldsymbol{k}, \omega ; \boldsymbol{k}^{\prime}, \omega^{\prime}\right)=\sum_{a} \frac{i e_{a}}{m_{a}} \frac{1}{\omega \omega^{\prime} \omega^{\prime \prime}} \\
\times\left[\omega \Gamma_{i l}^{(a)}(\omega) \Gamma_{\alpha p}^{(a)}\left(\omega^{\prime \prime}\right) k_{\alpha}^{\prime}-\omega^{\prime} \Gamma_{i l}^{(a)}\left(\omega^{\prime}\right) \Gamma_{s p}^{(a)}\left(\omega^{\prime \prime}\right) k_{s}\right. \\
+\omega \Gamma_{i p}^{(a)}(\omega) \Gamma_{\alpha l}^{(a)}\left(\omega^{\prime}\right) k_{\alpha}^{\prime \prime}-\omega^{\prime} \Gamma_{i j}^{(a)}(\omega) \Gamma_{p l}^{(a)}\left(\omega^{\prime}\right) k_{j}^{\prime \prime} \\
\left.-\omega^{\prime \prime} \Gamma_{i j}^{(a)}(\omega) \Gamma_{l p}^{(a)}\left(\omega^{\prime \prime}\right) k_{j}^{\prime}-\omega^{\prime \prime} \Gamma_{i p}^{(a)}\left(\omega^{\prime \prime}\right) \Gamma_{s l}^{(a)}\left(\omega^{\prime}\right) k_{s}\right],
\end{gathered}
$$

where $\Gamma_{i j}^{(a)}(\omega)=-g_{a}(\omega) \delta_{i j}+h_{a}(\omega) b_{i} b_{j}-i l_{a}(\omega) e_{i j l} b_{l}$.

It should be noted that at $\omega^{\prime \prime}=0 \quad\left(\omega=\omega^{\prime}\right)$ the tensor $S_{i p l}$ has a singularity, due to the adopted cold plasma model. Taking into account the thermal motion of the plasma particles the frequency change, in the scattering process, is on the order of $\sim k v_{T e}=\left(v_{T e} / c\right) \omega_{0}$, where $v_{T e}$ is the electron thermal velocity. We introduce the truncation parameter $T$, which is related to the frequency change $\omega^{\prime \prime}$ by the relation $\omega^{\prime \prime}=1 / T$. Obviously at $\omega=\omega^{\prime}$ we have $\omega_{0} T \sim c / v_{T e}$.

Let us consider the case when the test particle is at rest at a plane plasma-vacuum boundary. We consider the radiation escaping from the plasma into the vacuum due to the scattering (or transformation) of the magnetized plasma waves on this stationary particle. A more rigorous statement of the problem (boundary-value problem) requires that the generated surface waves are also taken into account. However, it should be emphasized that their intensity decays exponentially with distance from the boundary. Here we are interested only in the scattered bulk waves and the influence of the plasma boundary is neglected.

Consider Equation (9) for the intensity of the scattered radiation in the vacuum, where $\varepsilon_{i j}(\boldsymbol{k}, \omega) \rightarrow \delta_{i j}+i 0$. For the tensor $T_{i j}$ in this limit we obtain

$$
\begin{gathered}
T_{j i}\left(\boldsymbol{k}, \omega_{0}\right)-T_{i j}^{*}\left(\boldsymbol{k}, \omega_{0}\right) \\
=2 \pi i \frac{\omega_{0}^{2}}{c^{2}}\left(\delta_{i j}-n_{i} n_{j}\right) \delta\left(k^{2}-\omega_{0}^{2} / c^{2}\right),
\end{gathered}
$$


where $\boldsymbol{n}=\boldsymbol{k} / k$ is the unit vector in the direction of the wave vector $\boldsymbol{k}$ of the scattered waves. It thus follows from (16) that the scattering process occurs with no frequency change, while the wavelength differs from that of the incident waves $\left(k / k_{0}=\omega_{0} / k_{0} c=\eta\right)$ owing to the difference between the phase velocities of the plasma waves and the speed of light in a vacuum.

Using Equations (9), (15), and (16) as well as the relation $\varepsilon(\boldsymbol{k}, 0)=1+\left(k \lambda_{D}\right)^{-2}$ for the static dielectric function, where $\lambda_{D}$ is the Debye screening length of the plasma, for the total intensity of the scattered waves after lengthy but straightforward calculations we finally obtain

$$
W_{s}=\int I\left(\boldsymbol{n}, \boldsymbol{n}_{0}\right) d \Omega,
$$

where $d \Omega=\sin \theta d \theta d \varphi, \cos \theta=\boldsymbol{n} \cdot \boldsymbol{n}_{0}, \theta$ is the scattering angle, $\boldsymbol{n}_{0}=\boldsymbol{k}_{0} / k_{0}$ is the unit vector in the direction of the incident wave vector $\boldsymbol{k}_{0}$, and $I\left(\boldsymbol{n}, \boldsymbol{n}_{0}\right)$ is the angular distribution of the scattered radiation,

$$
\begin{gathered}
I\left(\boldsymbol{n}, \boldsymbol{n}_{0}\right)=\frac{I_{0} Z^{2}\left(\omega_{0} T\right)^{2} \Im\left(\boldsymbol{n}, \boldsymbol{n}_{0}\right)}{\left[\eta^{2}+1+\lambda^{2} / \lambda_{D}^{2}-2 \eta\left(\boldsymbol{n} \cdot \boldsymbol{n}_{0}\right)\right]^{2}} \\
\mathfrak{I}\left(\boldsymbol{n}, \boldsymbol{n}_{0}\right)=\sum_{a ; b} \mu_{a} \mu_{b} \Psi^{(a)}\left(\boldsymbol{n}, \boldsymbol{n}_{0}\right) \Psi^{(b)}\left(\boldsymbol{n}, \boldsymbol{n}_{0}\right) \\
\times \Phi_{a b}\left(\omega, \boldsymbol{n}, \boldsymbol{n}_{0}\right) \\
\Psi^{(a)}\left(\boldsymbol{n}, \boldsymbol{n}_{0}\right)=G_{a}\left(\eta \boldsymbol{n}-\boldsymbol{n}_{0}\right)^{2}+H_{a}\left[\eta(\boldsymbol{n} \cdot \boldsymbol{b})-\left(\boldsymbol{n}_{0} \cdot \boldsymbol{b}\right)\right]^{2} \\
\Phi_{a b}\left(\omega, \boldsymbol{n}, \boldsymbol{n}_{0}\right)=g_{a}(\omega) g_{b}^{*}(\omega)\left[1-|\boldsymbol{n} \cdot \boldsymbol{e}|^{2}\right] \\
+l_{a}(\omega) l_{b}^{*}(\omega)\left\{\left.[\boldsymbol{e} \times \boldsymbol{b}]\right|^{2}-|\boldsymbol{n} \cdot[\boldsymbol{e} \times \boldsymbol{b}]|^{2}\right\} \\
+h_{a}(\omega) h_{b}^{*}(\omega)|\boldsymbol{e} \cdot \boldsymbol{b}|^{2}\left[1-(\boldsymbol{n} \cdot \boldsymbol{b})^{2}\right] \\
+g_{a}(\omega) h_{b}^{*}(\omega)\left[(\boldsymbol{n} \cdot \boldsymbol{b})(\boldsymbol{n} \cdot \boldsymbol{e})(\boldsymbol{e} \cdot \boldsymbol{b})^{*}-|\boldsymbol{e} \cdot \boldsymbol{b}|^{2}\right] \\
+g_{b}^{*}(\omega) h_{a}(\omega)\left[(\boldsymbol{n} \cdot \boldsymbol{b})(\boldsymbol{n} \cdot \boldsymbol{e})^{*}(\boldsymbol{e} \cdot \boldsymbol{b})-|\boldsymbol{e} \cdot \boldsymbol{b}|^{2}\right] \\
+i g_{a}(\omega) l_{b}^{*}(\omega)\left[\boldsymbol{e} \cdot\left[\boldsymbol{b} \times \boldsymbol{e}^{*}\right]+(\boldsymbol{n} \cdot \boldsymbol{e})\left(\boldsymbol{n} \cdot\left[\boldsymbol{e}{ }^{*} \times \boldsymbol{b}\right]\right)\right] \\
+i g_{b}^{*}(\omega) l_{a}(\omega)\left[\boldsymbol{e} \cdot\left[\boldsymbol{b} \times \boldsymbol{e}^{*}\right]-(\boldsymbol{n} \cdot \boldsymbol{e})^{*}(\boldsymbol{n} \cdot[\boldsymbol{e} \times \boldsymbol{b}])\right] \\
+i(\boldsymbol{n} \cdot \boldsymbol{b})\left[(\boldsymbol{e} \cdot \boldsymbol{b})^{*}(\boldsymbol{n} \cdot[\boldsymbol{e} \times \boldsymbol{b}]) l_{a}(\omega) h_{b}^{*}(\omega)\right. \\
\left.\left.-\left[\boldsymbol{e}^{*} \times \boldsymbol{b}\right]\right) l_{b}^{*}(\omega) h_{a}(\omega)\right]
\end{gathered}
$$

In Equations (18)-(21) we have introduced the following notations: $\lambda=1 / k_{0}$ is the wavelength of the incident wave, $I_{0}=\left(c\left|\varepsilon_{0}\right|^{2} / 2 \pi\right) r_{0}^{2}, r_{0}=e^{2} / m c^{2}$ is the electron classical radius, and $G_{a}=-i g_{a}(0)$, $H_{a}=i h_{a}(0), \mu_{a}=m e_{a} / m_{a} e$.

Below, in Sections 3 and 4 , in the case of scattering and transformations of the high-frequency plasma waves we consider the interaction of the incident wave only with the electron component of the plasma omitting the index $a$ in expressions (19)-(21), assuming that the quantities $g(\omega), h(\omega), l(\omega), G$, and $H$ are related to the electrons. However, the ion component of the plasma must be taken into account in the case of low-frequency incident waves when $\omega_{0} \sim \omega_{c i}, \omega_{p i}$ (Sections 5 and 6). Furthermore, we consider the general expressions (18)-(21) in some special cases, assuming that the incident wave propagates perpendicular to the magnetic field B direction. We also assume that the incident wave propagates perpendicular to the plasma-vacuum interface (i.e., we choose the magnetic field to be parallel to the plasma boundary).

\section{Scattering of Ordinary Waves}

We first consider the scattering of ordinary waves from a stationary charged particle. It is well known [17] that an ordinary wave is a linearly polarized transverse $\left(\boldsymbol{E}_{0} \perp \boldsymbol{k}_{0}\right.$, where $\boldsymbol{E}_{0}=2 \boldsymbol{\varepsilon}_{0}$ ) electromagnetic wave propagating across a magnetic field. The polarization vector of this wave is parallel to the external magnetic field, $\boldsymbol{E}_{0} \| \boldsymbol{B}$, while its frequency is related to the wave vector by the usual dispersion equation for transverse electromagnetic waves propagating in a plasma, $\omega_{0}^{2}=\omega_{p}^{2}+k_{0}^{2} c^{2}$. The amplitude of the magnetic field of the incident wave is determined by the relation $\boldsymbol{B}_{0}=\left(c / \omega_{0}\right)\left[\boldsymbol{k}_{0} \times \boldsymbol{E}_{0}\right]$.

We introduce a spherical coordinate system with the polar $z$ axis in the direction of the vector $\boldsymbol{k}_{0}$ and the $y$ axis in the direction of the vectors $\boldsymbol{E}_{0}$ and $\boldsymbol{B}$ (Figure 1). The angle $\varphi$ is determined from the direction of the

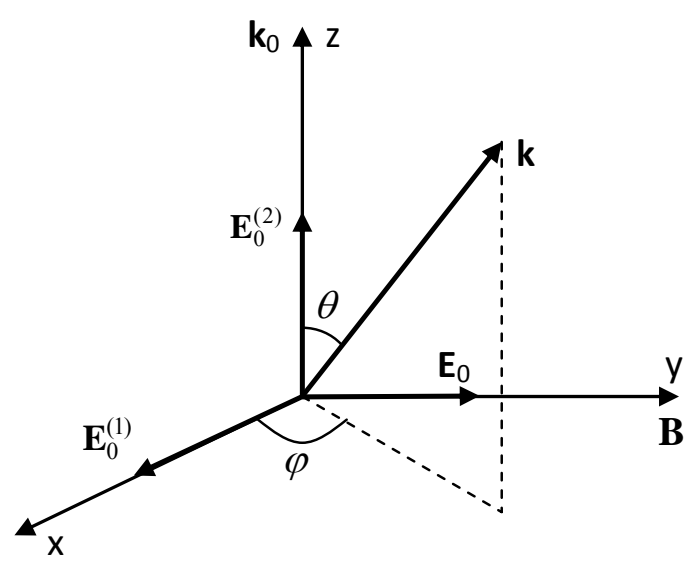

Figure 1. Diagram illustrating the scattering of an ordinary wave from a stationary charged particle located at the plane of a plasma-vacuum interface. The wave is traveling perpendicular to the plasma surface toward its boundary. The magnetic field is parallel to the interface and is directed along the polarization vector of the incident wave. 
$x$ axis. Then, taking into account the dispersion law for the incident ordinary wave and neglecting the ion component of the plasma, from (18) we obtain

$$
I(\theta, \varphi)=I_{0} \frac{Z^{2}\left(\omega_{0} T\right)^{2} \Psi^{2}(\theta, \varphi) \Phi\left(\omega_{0}, \theta, \varphi\right)}{\left(\eta^{2}+1+\lambda^{2} / \lambda_{D}^{2}-2 \eta \cos \theta\right)^{2}},
$$

where $\eta^{2}=1+\lambda^{2} / \lambda_{p}^{2}, \lambda_{p}=c / \omega_{p e}$,

$$
\begin{gathered}
\Psi(\theta, \varphi)=G\left(\eta^{2}+1-2 \eta \cos \theta\right)+H \eta^{2} \sin ^{2} \theta \sin ^{2} \varphi \\
\Phi(\omega, \theta, \varphi)=\frac{\omega_{p e}^{2}}{\omega^{2}+v^{2}}\left(1-\sin ^{2} \theta \sin ^{2} \varphi\right)
\end{gathered}
$$

and the angle $\theta$ varies in the range $0 \leq \theta \leq \pi / 2$. The collision frequency $v$ can be omitted from (24), since $v<<\omega_{0}$ for any $k_{0}$.

The wave vector of the scattered wave is easily determined by equating to zero the argument of the delta function in (16), $k=\omega_{0} / c>k_{0}$. This relation indicates that a long wavelength ordinary wave is transformed into short wavelength electromagnetic radiation in a vacuum.

Let us briefly consider the results which follow from (22) in the absence of a magnetic field ( $\left.H=0, G=\omega_{p e} / v\right)$. In this case and for $\lambda<\lambda_{p}$ the radiation is concentrated mainly in the direction perpendicular to the zy plane, i.e., the scattered wave escapes into the vacuum almost parallel to the vacuum-plasma interface. For scattering of a long waves $\left(\lambda>\lambda_{p}\right)$ the radiation is uniformly distributed (i.e., it does not depend on the angle $\theta$ ) in the $x z$ plane.

In the limit of wavelengths that are large compared to $\lambda_{p}$ the intensity of the scattered radiation does not depend on wavelength, in accordance with (22) and (23), and has the form

$$
\begin{aligned}
I(\theta, \varphi)= & I_{0} \tau^{-4} G^{2} Z^{2}\left(\omega_{p e} T\right)^{2}\left(1-\sin ^{2} \theta \sin ^{2} \varphi\right) \\
& \times\left(1+\frac{H}{G} \sin ^{2} \theta \sin ^{2} \varphi\right)^{2},
\end{aligned}
$$

where $\tau=\lambda_{p} / \lambda_{D}>>1$. From (25) it is seen that in the absence of a magnetic field $(H=0)$ the scattering occurs just as from a point charge $Z e$ (Thomson scattering) having an effective mass $m_{\text {eff }}=Z m \tau^{2} /\left(\omega_{p e} T G\right)$ [16]. Thus, the term proportional to $H$ in (25) determines the scattering of long waves due to plasma anisotropy.

It will be shown below that a sufficiently strong magnetic field $\left(\omega_{c e} \gg v\right.$ or $H \gg \rightarrow$ ) can significantly affect the scattering pattern observed in the absence of an external magnetic field. The angular distribution of the intensity of scattered radiation in this case has a maximum, the position of which is determined by the relation

$$
(\boldsymbol{n} \cdot \boldsymbol{b})^{2}=\sin ^{2} \theta \sin ^{2} \varphi \cong \frac{2-v^{2} / \omega_{c e}^{2}}{3} .
$$

From (26) it is seen that the maximum of the intensity exists only for sufficiently strong magnetic fields, $\omega_{c e}>v / \sqrt{2}$. In the opposite case with $\omega_{c e}<v / \sqrt{2}$ the intensity decreases monotonically, while for sufficiently small angle $\varphi\left(\sin ^{2} \varphi<\left(2-v^{2} / \omega_{c e}^{2}\right) / 3\right)$ but for $\omega_{c e}>v / \sqrt{2}$ it increases monotonically with $\theta$. From (25) and (26) it follows that the maximum of the intensity decreases slowly (by a factor of about 2.2) as the magnetic field increases from zero to the values of $\omega_{c e}>>\omega_{p e}$. The function $I(\theta, \varphi)$ is shown in Figure 2 for the scattering of long waves $\left(\lambda=4 \lambda_{p}\right)$ as a function of $\theta$ and $\varphi$. It is seen that the scattered radiation is concentrated mainly near a contour on $\theta, \varphi$ plane defined by (26). We also note that these equations define two cones $(\boldsymbol{n} \cdot \boldsymbol{b})^{2}=$ const with apices at the point $x=y=z=0 \quad$ (see Figure 1).

With a decrease of the incident wave wavelength the intensity of the scattered radiation increases rapidly, approximately as $\lambda^{-4}$ (see the denominator of (22)), up to $\lambda \sim \lambda_{D}$. Here the intensity has a maximum, the position of which is determined by Equation (26), in the wavelength range $\lambda_{D}<\lambda<\lambda_{p}$. It should be noted that the features of the angular distribution of the scattered waves discussed above for $\lambda>\lambda_{p}$ are retained in the case of small wavelengths.

In the limit of very short waves $\left(\lambda<\lambda_{D}\right)$ the angular distribution $I(\theta, \varphi)$ is changed significantly. Under the condition $\omega_{c e}>>v, \sin \varphi>v / \omega_{c e}$, for example, (22) takes the form

$$
\begin{aligned}
I(\theta, \varphi)=I_{0} Z^{2} & \left(\omega_{p e} T\right)^{2}\left[\frac{H \sin ^{2} \theta \sin ^{2} \varphi}{4 \sin ^{2}(\theta / 2)+\lambda^{2} / \lambda_{D}^{2}}\right]^{2} \\
& \times\left(1-\sin ^{2} \theta \sin ^{2} \varphi\right) .
\end{aligned}
$$

The intensity maximum is shifted toward smaller $\theta$ in this case, while the position of that maximum is determined by the equation

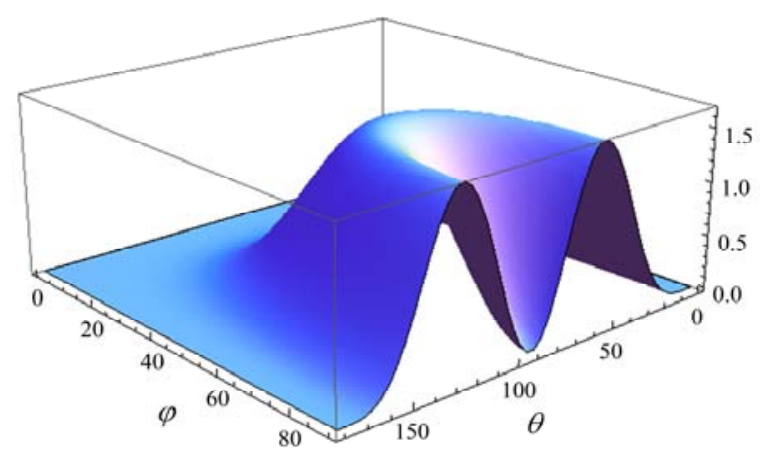

Figure 2. Angular distribution (normalized to $10^{-7} J_{0}$, where $\left.J_{0}=I_{0} Z^{2}\left(\omega_{p e} T\right)^{2}\right)$ of the scattered ordinary wave in a long wavelength range $\left(\lambda=4 \lambda_{p}\right)$. The calculations were done for $\tau=10^{2}, v / \omega_{p e}=0.1$, and $\omega_{c e} / \omega_{p e}=3$. 


$$
\theta_{\max } \cong\left[\left(2 \lambda / \lambda_{D}\right)\left(1+2 \sin ^{2} \varphi\right)^{-1 / 2}\right]^{1 / 2} .
$$

As follows from (27) and (28), the maximum intensity increases rapidly with increasing $\varphi$. In Figure 3 we demonstrate the angular distribution $I(\theta, \varphi)$ for the scattering of the short waves $\left(\lambda=0.1 \lambda_{D} \ll \lambda_{p}\right)$. Thus, the scattering in this case occurs mainly in the direction of propagation of the ordinary wave.

The total cross section for scattering from a stationary particle is obtained from (22) after integration over the angles $\theta$ and $\varphi$, where for ordinary waves from (12)-(14) we obtain $v_{g}=c /\left(1+\lambda^{2} / \lambda_{p}^{2}\right)^{1 / 2}$ and $S_{0} \cong v_{g} / c$. Since the general expression for the cross section is cumbersome, below we consider only some particular cases. In the case of scattering of very short waves $\left(\lambda<<\lambda_{D}\right)$ the cross section is almost constant and is given by

$$
\sigma(\lambda) \cong \sigma_{T} \frac{Z^{2}}{2}\left(\omega_{p e} T\right)^{2}\left(a_{1} G^{2}+b_{1} G H+c_{1} H^{2}\right)
$$

where $\sigma_{T}=(8 \pi / 3) r_{0}^{2}$ is the Thomson cross section, $a_{1}=1, \quad b_{1}=39 / 64, c_{1}=45 / 256$.

In the intermediate regime with $\lambda_{D}<\lambda<\lambda_{p}$ the cross section decreases as $\sigma(\lambda) \cong \sigma_{T} \sigma_{1}\left(\lambda_{p} / \lambda\right)^{4}$, where

$$
\sigma_{1}=\frac{11 Z^{2}}{20 \tau^{4}}\left(\omega_{p e} T\right)^{2}\left(a_{2} G^{2}+b_{2} G H+c_{2} H^{2}\right)
$$

with $a_{2}=1, b_{2}=17 / 44, c_{2}=6 / 77$.

In the case of long wavelengths $\left(\lambda>\lambda_{p}\right)$ the cross section increases linearly with the wavelength of the incident wave, $\sigma(\lambda) \cong \sigma_{T} \sigma_{2} \lambda / \lambda_{p}$, where

$$
\sigma_{2}=\frac{Z^{2}}{2 \tau^{4}}\left(\omega_{p e} T\right)^{2}\left(a_{3} G^{2}+b_{3} G H+c_{3} H^{2}\right)
$$

with $a_{3}=1, b_{3}=2 / 5, c_{3}=3 / 35$. Such a behavior of the cross section is explained by the fact that the incident

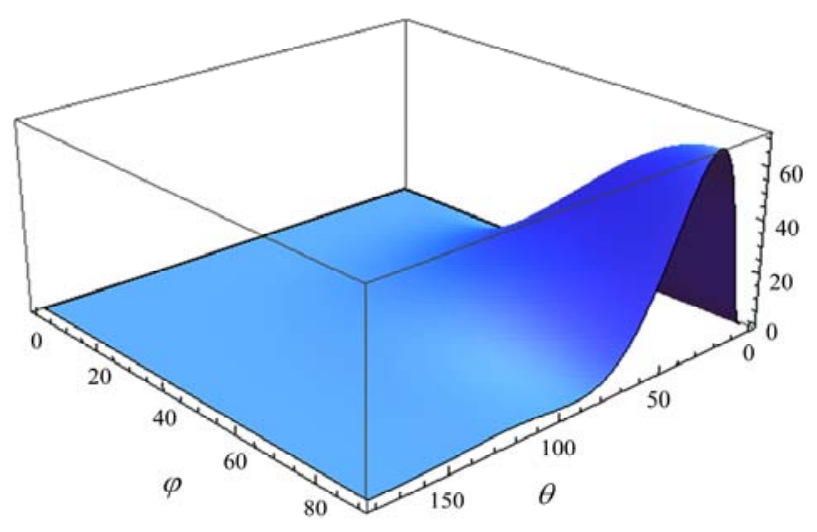

Figure 3. Angular distribution (normalized to $J_{0}$ ) of the scattered ordinary wave in a short wavelength range $(\lambda=$ $\left.10^{-3} \lambda_{p}\right)$. The values of the other parameters are the same as in Figure 2. and scattered waves have different group velocities, and for $\lambda>\lambda_{p}$ the energy flux in the incident wave is $S \sim 1 / \lambda$. Therefore, in the long wavelength range the total cross section does not coincide with the Thomson cross section for scattering from a point particle with a mass $m_{\text {eff }}$, as it occurs in the absence of a plasma boundary and an external magnetic field [18].

Using (30) and (31), the scattering cross section for ordinary waves at $\lambda>\lambda_{D}$ can be represented in the approximate form

$$
\sigma(\lambda) \cong \sigma_{T}\left[\sigma_{1}\left(\lambda_{p} / \lambda\right)^{4}+\sigma_{2} \lambda / \lambda_{p}\right] .
$$

From (32) it follows that at $\lambda_{\text {min }} \cong \lambda_{p}\left(4 \sigma_{1} / \sigma_{2}\right)^{1 / 5}$ the cross section has a minimum, the value of which is given by $\sigma_{\min } \cong 1.25 \sigma_{T}\left(4 \sigma_{1} \sigma_{2}^{4}\right)^{1 / 5}$.

The dependence of the scattering cross section on the magnetic field can be traced from (29)-(32). The cross section decreases monotonically with increasing magnetic field. This behavior is especially pronounced for $\lambda>\lambda_{D}$ and is one order of magnitude over the range of variation of the magnetic field from zero to $\beta=\omega_{c e} / \omega_{p e}>1$. The decrease of the cross section is due to the reduction of the transverse cyclotron motion of plasma electrons with the magnetic field. In the limit of very strong magnetic fields $(\beta>>1)$ the plasma behaves like a one-dimensional fluid, the motion of which is confined to oscillations along magnetic field lines.

\section{Transformation of Extraordinary Waves}

In this section we consider an extraordinary incident wave with a complex amplitude $\boldsymbol{\varepsilon}_{0}=(1 / 2)\left(\boldsymbol{E}_{0}^{(1)}-i \boldsymbol{E}_{0}^{(2)}\right)$ (where $\boldsymbol{E}_{0}^{(1)}$ and $\boldsymbol{E}_{0}^{(2)}$ are the real amplitudes), propagating across the magnetic field. It is well known [17] that in general an extraordinary wave is elliptically polarized in the $x y$ plane (Figure 1), i.e., $\boldsymbol{\varepsilon}_{0} \perp \boldsymbol{B}$. With no loss of generality, we choose the vectors $\mathbf{E}_{0}^{(1)}$ and $\boldsymbol{E}_{0}^{(2)} \quad$ such that $E_{0 \mathrm{x}}^{(1)}>0$ and $E_{0 \mathrm{y}}^{(1)}=E_{0 \mathrm{z}}^{(1)}=E_{0 \mathrm{x}}^{(2)}$ $=E_{0 \mathrm{y}}^{(2)}=0$ (Figure 1). For this choice, the amplitude of the magnetic field of the incident wave is determined by the relation $\boldsymbol{B}_{0}=\left(c / 2 \omega_{0}\right)\left[\boldsymbol{k}_{0} \times \boldsymbol{E}_{0}^{(1)}\right]$ and is directed along the external magnetic field.

The relation between the components $E_{0 \mathrm{x}}^{(1)}$ and $E_{0 \mathrm{z}}^{(2)}$ is given by the equation [17]

$$
\frac{E_{0 \mathrm{z}}^{(2)}}{E_{0 \mathrm{x}}^{(1)}}=\frac{\varepsilon_{3}\left(\omega_{0}\right)}{\varepsilon_{1}\left(\omega_{0}\right)} \equiv P\left(\omega_{0}\right),
$$

while the relation between the frequency and the wave vector is given by the dispersion equation for the extraordinary waves [17],

$$
k_{0}^{2}=\frac{\omega_{0}^{2}}{c^{2}} \frac{2 \varepsilon_{R}\left(\omega_{0}\right) \varepsilon_{L}\left(\omega_{0}\right)}{\varepsilon_{R}\left(\omega_{0}\right)+\varepsilon_{L}\left(\omega_{0}\right)}
$$


with $\varepsilon_{R}(\omega)=\varepsilon_{1}(\omega)-\varepsilon_{3}(\omega)$ and $\varepsilon_{L}(\omega)=\varepsilon_{1}(\omega)+\varepsilon_{3}(\omega)$.

The energy flux of the incident wave and the intensity of the scattered extraordinary waves are determined by the expressions $\boldsymbol{S}=\left(c\left|\boldsymbol{E}_{0}\right|^{2} / 2 \pi\right) \boldsymbol{S}_{0}$, (14), and (18)-(21), respectively, where

$$
\begin{gathered}
\boldsymbol{S}_{0}=\frac{\boldsymbol{v}_{g}}{2 \omega_{0} c} \frac{\partial}{\partial \omega_{0}}\left[\omega_{0}^{2} \varepsilon_{1}\left(\omega_{0}\right) \frac{1+3 P^{2}\left(\omega_{0}\right)}{1+P^{2}\left(\omega_{0}\right)}\right], \\
\Phi(\omega, \theta, \varphi)=q(\omega)\left[1-\sin ^{2} \theta \cos ^{2} \varphi+Q(\omega) \sin ^{2} \theta\right],(36) \\
q(\omega)=\frac{\omega_{p e}^{2}\left(\omega^{2}-\omega_{p e}^{2}\right)^{2}}{\omega^{2}\left(\omega^{2}-\omega_{H}^{2}\right)^{2}+\omega_{p e}^{4} \omega_{c e}^{2}}, Q(\omega)=\frac{\omega^{2} \omega_{c e}^{2}}{\left(\omega^{2}-\omega_{p e}^{2}\right)^{2}} .
\end{gathered}
$$

We investigate the expressions obtained for $I(\theta, \varphi)$ for high-frequency (electron) extraordinary waves. The ion component of the plasma can be again neglected in this case. The two solutions of the dispersion equation (34) then have the form [17]

$$
\omega_{0}^{( \pm)}\left(k_{0}\right)=2^{-1 / 2}\left[f_{1}\left(k_{0}\right) \pm \sqrt{f_{1}^{2}\left(k_{0}\right)-4 f_{2}\left(k_{0}\right)}\right]^{1 / 2}
$$

where $f_{1}\left(k_{0}\right)=\omega_{1}^{2}+\omega_{2}^{2}+k_{0}^{2} c^{2}, f_{2}\left(k_{0}\right)=\omega_{1}^{2} \omega_{2}^{2}+\omega_{H}^{2} k_{0}^{2} c^{2}$, and $\omega_{H}^{2}=\omega_{c e}^{2}+\omega_{p e}^{2}$ is the upper hybrid frequency. The quantities $\omega_{1}$ and $\omega_{2}$ are the cutoff frequencies which are the solutions of the equations $\varepsilon_{L}\left(-\omega_{2}\right)=\varepsilon_{L}\left(\omega_{1}\right)=0$ and $\varepsilon_{R}\left(-\omega_{1}\right)=\varepsilon_{R}\left(\omega_{2}\right)=0$, respectively, and under the condition $\omega_{c e} \omega_{c i} \ll \omega_{p e}^{2}$ (which is fully justified for both laboratory and astrophysical conditions) have the form [17]

$$
\omega_{2}=\omega_{c e} / 2+\sqrt{\omega_{c e}^{2} / 4+\omega_{p e}^{2}}, \quad \omega_{1}=\omega_{p e}^{2} / \omega_{2}<\omega_{2} .
$$

In this section we consider only the scattering of the high-frequency mode $\omega_{0}^{(+)}$. We briefly recall (see also (38)) that for this mode $\omega_{0}^{(+)}\left(k_{0}\right)$ increases monotonically from $\omega_{0}^{(+)}\left(k_{0}\right)=\omega_{2}$ at $k_{0} \rightarrow 0 \quad$ to $\omega_{0}^{(+)}\left(k_{0}\right)=k_{0} c$ at $k_{0} \rightarrow \infty$. Since $P\left(\omega_{0}\right)>0$ (or $\left.E_{0 \mathrm{z}}^{(2)}>0\right)$ in this frequency range the high-frequency wave has, in general, right-hand elliptic polarization in the $x z$ plane (in the positive $y$ direction). In the case of long waves $\left(k_{0} \rightarrow 0\right), P\left(\omega_{2}\right)=1$, the wave is almost circularly polarized, whereas in the case of short waves $\left(k_{0} \rightarrow \infty\right), P\left(\omega_{0}\right) \ll 1$, this mode consists of a linearly polarized, transverse electromagnetic wave. In the latter case the only difference between an extraordinary and an ordinary wave is that the polarization vector of an extraordinary wave is perpendicular to the external magnetic field.

The wave vector of the scattered wave is determined by the expression $k=\omega_{0} / c$. From (38) we conclude that $\omega_{0} / c>k_{0}$ in the entire wavelength range of the incident wave. Thus, as in the case of an ordinary wave, the transformation of extraordinary waves into electromagnetic radiation in a vacuum is accompanied by a decrease of the wavelength.

Let us consider the angular distribution of the scattered waves in the limits of small and large $\lambda$. In the limit of very short waves $\left(\lambda \ll \lambda_{D}\right)$ the angular distribution has a maximum at the values of the small angle $\theta$ determined by (28), in which $\sin \varphi$ is replaced by $\cos \varphi$. All the properties obtained for ordinary waves in the range of $\lambda$ under consideration are retained in this case. In this limit the cross section is almost constant and is determined by (29) in which the numerical coefficients are $a_{1}=1, b_{1}=61 / 64, c_{1}=381 / 1280$.

In the intermediate wavelength range with $\lambda_{D}<\lambda \ll c \alpha / \omega_{H}$, where $\alpha$ is a number on the order of unity, the intensity of the scattered radiation decreases rapidly with increasing $\lambda$ as $\lambda^{-4}$. The angular distribution of the scattered waves is also changed. The intensity maximum is shifted toward larger angles $\theta$, and for $\omega_{c e} \gg v, \sin \varphi>v / \omega_{c e}$, and $\cos ^{2} \varphi>2 / 3$ the position of that maximum is determined by the expression $\sin ^{2} \theta \cos ^{2} \varphi \cong 2 / 3$. However, $I(\theta, \varphi)$ increases monotonically with further increasing $\varphi\left(\cos ^{2} \varphi<2 / 3\right)$ and reaches the maximum value at $\theta \cong \varphi \cong \pi / 2$ (or $\varphi \cong 3 \pi / 2$ ) (Figure 4). In the same wavelength range the scattering cross section has the form $\sigma(\lambda) \cong \sigma_{T} \sigma_{1}\left(\lambda_{p} / \lambda\right)^{4}$, where $\sigma_{1}$ is determined from (30) with coefficients $a_{2}=1, b_{2}=39 / 44$, and $c_{2}=18 / 77$.

In accordance with (22), (23), (36), and (37) in the limit of the wavelengths larger than $c / \omega_{c e}$, the intensity of the scattered radiation is

$$
\begin{gathered}
I(\theta, \varphi)=I_{0} Z^{2}\left(\omega_{p e} T\right)^{2} \frac{f_{0}^{8}(\beta)}{2 \tau^{4}}\left(1+\sin ^{2} \theta \sin ^{2} \varphi\right) \\
\times\left(G+H \sin ^{2} \theta \sin ^{2} \varphi\right)^{2}, \\
f_{0}(\beta) \equiv \omega_{2} / \omega_{p e}=\beta / 2+\sqrt{1+\beta^{2} / 4} .
\end{gathered}
$$

It is seen from Equation (40) that the intensity of the scattered radiation increases monotonically with $(\boldsymbol{n} \cdot \boldsymbol{b})=\sin \theta \sin \varphi$ and scattering occurs mainly in the direction of the external magnetic field.

In the limit of $\lambda \gg c / \omega_{c e}$, from (35) we obtain $S_{0}=\left(\lambda_{p} / \lambda\right) F_{1}(\beta)$, where

$$
F_{1}(\beta)=\frac{1}{f_{0}^{2}(\beta) \sqrt{4+\beta^{2}}} \frac{f_{0}(\beta)\left(1+4 \beta^{2}\right)+5 \beta / 2}{f_{0}(\beta)\left(1+4 \beta^{2}\right)+3 \beta} .
$$

Then the cross section reads $\sigma(\lambda) \cong \sigma_{T} \sigma_{2} \lambda / \lambda_{p}$, where $\sigma_{2}$ is determined from (31) with coefficients $a_{3}=f_{0}^{8}(\beta) / F_{1}(\beta), b_{3}=4 a_{3} / 5$, and $c_{3}=9 a_{3} / 35$. 


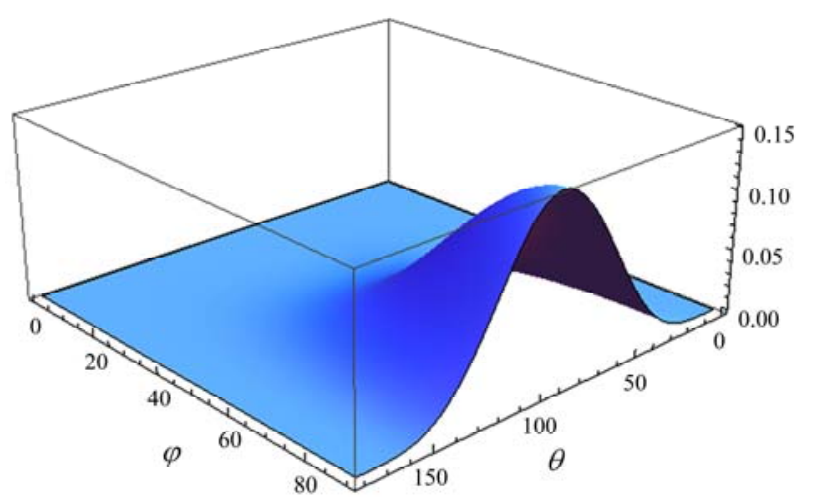

Figure 4. Angular distribution (normalized to $\boldsymbol{J}_{\mathbf{0}}$ ) of a scattered extraordinary wave with a frequency $\omega_{0}^{(+)}$in the intermediate wavelength range $\left(\lambda=5 \lambda_{D}\right)$. The values of the other parameters are the same as in Figure 2.

From these expressions and (40)-(42) it follows that at $\lambda \gg c / \omega_{c e}$ and $\beta \gg 1$ the angular distribution and the scattering cross section of the extraordinary waves are proportional to $\beta^{8}$ and $a_{3}=\beta^{11}$, respectively, and increase considerably with the magnetic field. At $\lambda \gg c / \omega_{c e}$ an extraordinary wave has right-hand circular polarization in the $x z$ plane and at $\beta \gg 1$ its frequency is $\omega_{2} \approx \omega_{c e}$. Thus in this case a specific cyclotron resonance may occur which, however, differs from the usual one so that the incident wave is polarized in the plane of incidence and propagates across the external magnetic field [17].

\section{Transformation of Waves of Intermediate Frequency}

In this section we consider the transformation of the extraordinary waves with a frequency spectrum $\omega_{0}^{(-)}\left(k_{0}\right)$ determined by (38). The frequency $\omega_{0}^{(-)}\left(k_{0}\right)$ increases monotonically (see (38)) from $\omega_{0}^{(-)}=\omega_{1}$ at $k_{0} \rightarrow 0$ to $\omega_{0}^{(-)}=\omega_{H}$ at $k_{0} \rightarrow \infty$. Since the frequency of this mode is high compared to the characteristic ionic frequencies (see, e.g., [17]), we neglect here the contribution of plasma ions both in the scattering current and in the wave dispersion. From (33) it follows that in this frequency range $P\left(\omega_{0}\right)<0$ (or $E_{0 z}^{(2)}<0$ ), i.e., this mode, in general, has left-hand elliptical polarization in the $x z$ plane (Figure 1) and cannot resonate with plasma electrons. In the case of long waves $\left(k_{0} \rightarrow 0\right), P\left(\omega_{1}\right)=-1$, and the wave is almost circularly polarized, whereas in the case of short waves $\left(k_{0} \rightarrow \infty\right), P\left(\omega_{H}\right) \rightarrow-\infty$, this mode consists of a longitudinal wave (upper-hybrid oscillations). In the latter case the upper-hybrid waves are transformed into electromagnetic radiation in a vacuum.

From (38) for $\omega_{0}^{(-)}$one concludes that $\omega_{0} / c \leq k_{0}$ at $\lambda \leq \lambda_{p}$ and $\omega_{0} / c>k_{0}$ at $\lambda>\lambda_{p}$. Thus, at $\lambda \leq \lambda_{p}$ and $\lambda>\lambda_{p}$ the scattering of the mode $\omega_{0}^{(-)}$is accom- panied by an increase or a decrease of the wavelength, respectively.

General expressions for the angular distribution of the scattered extraordinary waves have been obtained in Sections 3 and 4 (Equations (22), (36), and (37)). In the range of very short wavelengths $\left(\lambda \ll \lambda_{D}\right)$, from these expressions we obtain the angular distribution of the transformation of upper-hybrid oscillations,

$$
\begin{aligned}
& I(\theta, \varphi)=I_{0} Z^{2}\left(\omega_{p e} T\right)^{2} G^{2}\left(1+\beta^{2}\right) \\
& \quad \times\left[\beta^{2}+\sin ^{2} \theta\left(\beta^{2} \sin ^{2} \varphi+1\right)\right] .
\end{aligned}
$$

The transformation cross section in this wavelength range it is obtained from (35) and (43). After integration of Equation (43) with respect to the angles, one obtains $\sigma \cong \sigma_{T} \sigma_{0}\left(\lambda_{p} / \lambda\right)^{3}$, where

$$
\sigma_{0}=\frac{Z^{2}}{3}\left(\omega_{p e} T\right)^{2} G^{2} \sqrt{1+\beta^{2}}\left(1+\frac{1}{2 \beta^{2}}\right) .
$$

From this expression for the cross section it is seen that, in contrast to the scattering (transformation) of high-frequency waves, in which the cross section for $\lambda \ll \lambda_{D}$ is constant, in the case of intermediate upper-hybrid waves the cross section increases essentially (as $\lambda^{-3}$ ) with decreasing the wavelength of the incident wave. This feature is due to the strong reduction of the energy flux $\left(S_{0} \sim \lambda^{3}\right)$ in the incident wave.

Consider now the opposite limiting case of the long wavelengths, $\lambda \gg \lambda_{p}$. We first note that for sufficiently strong magnetic fields, $\omega_{c e}>\omega_{p e} / \sqrt{2}$ the frequency of these waves at $\lambda=\lambda_{c}=c /\left(2 \omega_{c e}^{2}-\omega_{p e}^{2}\right)^{1 / 2}$ coincides with the electron cyclotron frequency, $\omega_{0}^{(-)}=\omega_{c e}$. On the other hand, $P(\omega)=-1$ at $\omega=\omega_{c e}$ and the incident wave is circularly polarized in the $x z$ plane (see Figure 1). Near the cyclotron frequency, $\omega_{0} \approx \omega_{c e}$, the energy flux of the intermediate wave has the form

$$
S_{0} \sim \frac{2 \omega_{c e} \omega_{p e}^{4} \sqrt{2 \omega_{c e}^{2}-\omega_{p e}^{2}}}{\omega_{H}^{2}\left(\omega_{0}^{2}-\omega_{c e}^{2}\right)^{2}}
$$

and increases strongly due to the cyclotron resonance. This resonance is stabilized taking into account the electron-ion collisions. Here the energy flux can be very large but finite quantity. Thus, at $\lambda=\lambda_{c}$ the transformation cross section is vanishingly small, $\sigma \approx 0$.

In the limit of the long wavelengths, for the angular distribution from the general expressions (22) and (36) we obtain

$$
\begin{gathered}
I(\theta, \varphi)=\frac{I_{0} Z^{2}\left(\omega_{p e} T\right)^{2}}{2 \tau^{4} f_{0}^{8}(\beta)}\left(G+H \sin ^{2} \theta \sin ^{2} \varphi\right)^{2} \\
\quad \times\left(1+\sin ^{2} \theta \sin ^{2} \varphi\right) .
\end{gathered}
$$


In this limit $S_{0}=\left(\lambda_{p} / \lambda\right) F_{2}(\beta)$, where

$F_{2}(\beta)=f_{0}(\beta)\left\{\frac{2 f_{0}(\beta)}{\sqrt{4+\beta^{2}}}\left[1+\frac{\beta^{2}}{\left(2-f_{0}^{2}(\beta)\right)^{2}}\right]-\frac{1}{2}\right\}$.

The cross section in the limit $\lambda \gg \lambda_{p}$ is determined from the expression $\sigma(\lambda) \cong \sigma_{T} \sigma_{2} \lambda / \lambda_{p}$, where $\sigma_{2}$ is given by (31) with $a_{3}=1 / f_{0}^{8}(\beta) F_{2}(\beta), b_{3}=4 a_{3} / 5$, and $c_{3}=9 a_{3} / 35$. A comparison of these expressions for the angular distribution and the cross section with the similar expressions obtained in the case of the transformation of a high-frequency extraordinary wave shows that in the former case a strong external magnetic field can strongly suppress the transformation of an intermediate wave, the intensity of which decreases as $\beta^{-8}$ with increasing of the external magnetic field (see (46)).

From (1) and (4) it is seen that the intensity of the transformation of an intermediate wave increases monotonically with $\theta$ and takes a maximum value at $\theta=\pi / 2$ and $\varphi=\pi / 2$ (or $\varphi=3 \pi / 2$ ). Therefore, the radiation mainly escapes from the plasma parallel to its boundary in the direction of the external magnetic field (Figure 1).

The intensity $I(\theta, \varphi)$ of the transformation of the intermediate wave as a function of the wavelength and the angle $\theta$ is shown in Figure 5. From this figure it is seen that the intensity has a maximum in the short-wavelength range, whereas the intensity of the scattering (transformation) of the high-frequency waves decreases monotonically with wavelength of the incident wave.

At the end of this section we note that the restriction $v_{g}>v_{T e}$ on the group velocity (see Section 2 ) leads to a limitation $\lambda<\left(c / v_{T e}\right) \lambda_{p}\left(4+\beta^{2}\right)^{-1 / 2} / f_{0}^{2}(\beta)$ of the wavelength of the incident wave.

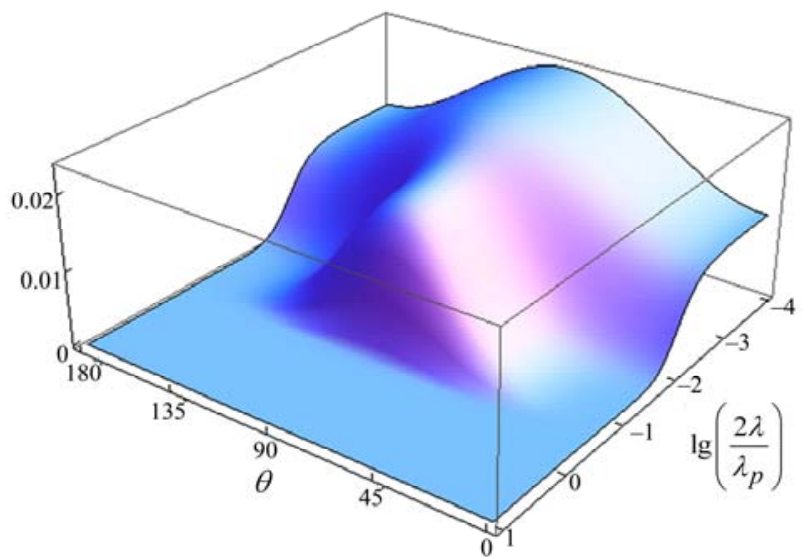

Figure 5. Dependence of the intensity $I(\theta, \varphi)$ (normalized to $J_{0}$ ) for a transformed intermediate wave on the wavelength and the angle $\theta$ for $\varphi=\pi / 2$. The values of the other parameters are the same as in Figure 2.

\section{Scattering of Low-Frequency Waves}

In this section we consider the scattering (transformation) of low-frequency magnetosonic and lower-hybrid plasma waves, the frequencies of which are much lower than the characteristic electron frequencies $\left(\omega_{c e}\right.$ and $\left.\omega_{p e}\right)$ and are comparable in order of magnitude with the ion-cyclotron and Langmuir frequencies $\omega_{c i}$ and $\omega_{p i}$, respectively. In this low-frequency limit one must take into account the dynamics of the plasma ions and their partial contributions to the dispersion equation and the scattering current.

From the general relation (34) we obtain an expression for the frequency of the low-frequency waves (see also [17]),

$$
\omega_{0}^{2}\left(k_{0}\right)=\omega_{L H}^{2} \frac{k_{0}^{2} u_{A}^{2}}{k_{0}^{2} u_{A}^{2}+\omega_{L H}^{2}},
$$

where $\omega_{L H}^{2}=\omega_{c e} \omega_{c i} \omega_{p e}^{2} / \omega_{H}^{2}$ is the lower hybrid frequency, $u_{A}=V_{A} / \sqrt{1+V_{A}^{2} / c^{2}}$, and $V_{A}$ is the Alfvén velocity.

From (48) it follows that $P\left(\omega_{0}\right)>0$ (or $E_{0 z}^{(2)}>0$ ), i.e., this mode in general has right-hand elliptical polarization in the $x z$ plane (Figure 1) and can resonate with plasma ions. In the case of the long magnetosonic waves $\left(k_{0} \rightarrow 0\right.$ ) we obtain $P(0)=0$ and the wave has transverse polarization, while in the case of short lower-hybrid waves $\left(k_{0} \rightarrow \infty\right), P\left(\omega_{L H}\right) \rightarrow \infty$ and this mode consists of a longitudinal wave. In the latter case we have the transformation of the lower-hybrid waves into electromagnetic radiation in a vacuum.

From the expression for $\omega_{0}$ it follows that $\omega_{0} / c<k_{0}$ for any $\lambda$. The transformation of the low-frequency mode is therefore accompanied by an increase in wavelength. Let us make some estimates. In astrophysical conditions for a density $\rho=10^{6} \mathrm{~g} / \mathrm{cm}^{3}$ and a magnetic field $B=10^{9} \mathrm{kG}$ we obtain that the transformation of the magnetosonic wave in a vacuum generates radiation with a wavelength exceeding that of the incident wave by two orders of magnitude, $k_{0} c / \omega_{0}=c / u_{\mathrm{A}} \cong 106$.

Consider now Equations (18)-(21) for the intensity of the transformation of the low-frequency waves. Taking into account the dynamics of the plasma ions (19)-(21) become

$$
\begin{gathered}
\Im(\omega, \theta, \varphi)=\Psi^{(e) 2}(\theta, \varphi) \Phi_{e e}(\omega, \theta, \varphi) \\
+2 \mu \Psi^{(e)}(\theta, \varphi) \Psi^{(i)}(\theta, \varphi) \Phi_{e i}(\omega, \theta, \varphi) \\
+\mu^{2} \Psi^{(i) 2}(\theta, \varphi) \Phi_{i i}(\omega, \theta, \varphi), \\
\Psi^{(a)}(\theta, \varphi)=G_{a}\left(\eta^{2}+1-2 \eta \cos \theta\right)+\eta^{2} H_{a} \sin ^{2} \theta \sin ^{2} \varphi,
\end{gathered}
$$


$\Phi_{a b}(\omega, \theta, \varphi)=q_{a b}(\omega)\left[1-\sin ^{2} \theta \cos ^{2} \varphi+Q_{a b}(\omega) \sin ^{2} \theta\right]$,

$q_{a b}(\omega)=\frac{\left[g_{a}(\omega)-l_{a}(\omega) P(\omega)\right]\left[g_{b}(\omega)-l_{b}(\omega) P(\omega)\right]}{1+P^{2}(\omega)}$,

$Q_{a b}(\omega)=\frac{\left[l_{a}(\omega)-g_{a}(\omega) P(\omega)\right]\left[l_{b}(\omega)-g_{b}(\omega) P(\omega)\right]}{\left[g_{a}(\omega)-l_{a}(\omega) P(\omega)\right]\left[g_{b}(\omega)-l_{b}(\omega) P(\omega)\right]}$.

In (50)-(53) the indices $a$ and $b$ take the values $e$ or $i$ to denote the contributions of plasma electrons and ions, $\mu=\mu_{i}=Z_{i} m / m_{i} \ll 1$, where $Z_{i}, m_{i}$, and $m$ are the charge number and mass of an ion and the electron mass, respectively, and the quantities $G_{a}, H_{a}, g_{a}(\omega)$, $l_{a}(\omega)$, and $P(\omega)$ are determined by (13) and (33), respectively.

In the limit of short wavelengths $\left(\lambda \ll \lambda_{D}\right)$, from (49)-(53) we obtain the angular distribution of the transformation of lower-hybrid oscillations,

$$
\begin{gathered}
I(\theta, \varphi)=I_{0} Z^{2}\left(\omega_{p e} T\right)^{2} \frac{\mu}{1+\beta^{2}} \\
\times\left[G_{1}^{2}\left(1-\sin ^{2} \theta \cos ^{2} \varphi\right)+\frac{\mu}{1+\beta^{2}} G_{2}^{2} \sin ^{2} \theta\right],
\end{gathered}
$$

where

$$
\begin{aligned}
& G_{1}=G_{e}+\mu^{3 / 2}\left(1+\beta^{2}\right) G_{i}, \\
& G_{2}=G_{e}-\mu^{1 / 2}\left(1+\beta^{2}\right) G_{i} .
\end{aligned}
$$

For small external magnetic fields $(\beta \ll 1)$ the contribution of the ions in (54) and (55) is negligible. For $\beta \gg 1$, however, the transformation occurs mainly due to the ionic current.

From (54) we derive the cross section for the transformation of lower-hybrid waves by integrating the latter over angles. The result is given by the expression $\sigma \cong \sigma_{T} \sigma_{0}\left(\lambda_{p} / \lambda\right)^{3}$, where

$$
\sigma_{0}=\frac{Z^{2}}{6}\left(\omega_{p e} T\right)^{2} \frac{\beta \sqrt{\mu}}{\sqrt{1+\beta^{2}}}\left(G_{1}^{2}+\frac{\mu}{1+\beta^{2}} G_{2}^{2}\right) .
$$

From (35) and (48) it follows that for the wavelengths $\lambda \cong \lambda_{c}=u_{A} / \omega_{c i}$ the frequency is close to the ion-cyclotron frequency $\omega_{0} \cong \omega_{c i}$. At these frequencies we obtain $P\left(\omega_{c i}\right)=1$. Therefore, near $\omega_{c i}$ the wave has right-hand circular polarization and, as noted above, it can resonate with plasma ions. Ion-cyclotron resonance occurs in this case and the energy flux of the incident wave increases sharply as

$$
S_{0} \sim \frac{u_{A}}{c} \frac{2 \omega_{c i}^{2} \omega_{p i}^{2}}{\left(\omega_{0}^{2}-\omega_{c i}^{2}\right)^{2}} .
$$

The cross section of the process thus approaches to zero at $\lambda \cong \lambda_{c}$.

In the limit of long magnetosonic waves $\left(\lambda \gg \lambda_{p}\right.$, $\lambda_{c}$ ), from (18) and (49)-(53) we obtain

$$
\begin{array}{r}
I(\theta, \varphi)=I_{0} Z^{2}\left(\omega_{p e} T\right)^{2}\left(\mu / \tau^{4}\right)\left(\lambda_{p} / \lambda\right)^{6} \sin ^{2} \theta \\
\times\left[G_{0}\left(\eta_{A}^{2}+1-2 \eta_{A} \cos \theta\right)+H_{0} \eta_{A}^{2} \sin ^{2} \theta \sin ^{2} \varphi\right],
\end{array}
$$

where $H_{0}=H_{e}-H_{i} \sqrt{\mu}, G_{0}=G_{e}-G_{i} \sqrt{\mu}, \quad \eta_{A}=u_{A} / c$. It should be noted that in the second term in (58) the contribution of ions can be neglected for any values of the magnetic field $\left(H_{e} \gg H_{i} \sqrt{\mu}\right)$, whereas in the first term the contribution of ions can be neglected only for weak external magnetic fields.

From (58) we find the cross section for the transformation of magnetosonic waves. After evaluation of the integrals over angles, we find $\sigma \cong \sigma_{T} \sigma_{1}\left(\lambda_{p} / \lambda\right)^{6}$, where

$$
\sigma_{1}=Z^{2}\left(\omega_{p e} T\right)^{2} \frac{\beta \mu^{3 / 2}}{2 \tau^{4}}\left[G_{0}\left(\eta_{A}^{2}+1-\frac{3}{4} \eta_{A}\right)+\frac{2}{5} H_{0} \eta_{A}^{2}\right]
$$

From (54) and (58) it is seen that the intensity of the transformation of the magnetosonic waves increases monotonically with angles $\theta$ and $\varphi$, approaching the maximum value at $\theta=\pi / 2$ and $\varphi=\pi / 2$ (or $\varphi=3 \pi / 2$ ). Consequently, as in the case of an intermediate wave, radiation escapes from the plasma mainly parallel to its boundary in the direction of the external magnetic field.

In Figure 6 we demonstrate the dependence of the intensity of transformation of a low-frequency wave as a function of wavelength and the angle $\theta$. From (54), (58), and Figure 6 it is seen that the efficiency of transformation of lower-hybrid waves far exceeds the efficiency of transformation of magnetosonic waves. However, the intensity of the emission produced by the transformation of magnetosonic waves can be essential if we take into account that it is proportional to the (large) quantities $Z^{2}$ and $T^{2}$. We note that the estimate of the cutoff parameter $T$ depends on the specific model of magnetized plasma.

\section{Discussion and Conclusions}

In this paper, we have presented a detailed investigation of the scattering and transformation of the plasma waves on heavy charged particle in magnetized plasma. The basic idea of this paper is that the scattering (transformation) occurs due to the nonlinear interaction of the incident wave with the polarization cloud surrounding the particle. In the course of this study we have derived some analytical results for the angular distribution and the cross section of the scattered (transformed) radiation and 


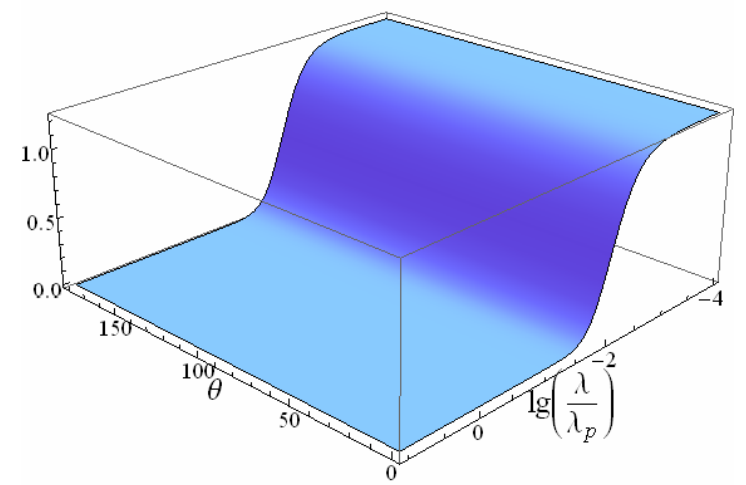

Figure 6. Dependence of $I(\theta, \varphi)$ (normalized to $\left.10^{-10} J_{0}\right)$ for a transformed low-frequency wave on the wavelength and the angle $\theta$ for $\varphi=\pi / 2$ and $\mu=10^{-5}$. The values of the other parameters are the same as in Figure 2.

we have shown that the problem is reduced to the determination of the nonlinear (three index) dielectric tensor of magnetized plasma.

After introduction to the general theory in Section 2, we have studied some particular cases of the scattering and transformation processes assuming that the incident wave propagates in the direction transverse to the external magnetic field. The angular distribution and the cross section for the scattering and transformation of high-frequency ordinary and extraordinary waves and low-frequency upper-hybrid, low-hybrid, and magnetosonic waves have been investigated within a cold plasma model which is valid when the group velocities of the incident and scattered waves exceed the thermal velocities of the plasma particles. A number of limiting and asymptotic regimes of short and long wavelengths have been studied. The theoretical expressions for the angular distribution of the scattered waves derived in this paper lead to a detailed presentation of a collection of data through figures.

We expect our theoretical model to be useful in experimental investigations of the wave scattering by plasma as well as in some astrophysical applications. Going beyond the presented model calculations which are based on the cold plasma approximation we can envisage a number of avenues. One of the improvements of our model will be to include the thermal effects which are particularly important in the case of dusty plasmas [15]. Furthermore, the theoretical model developed here although is strong but is not adopted for immediate astrophysical applications. For this purpose it is required 1) fully relativistic fluid calculations with appropriate equation of states and transport coefficients of a strongly magnetized and dense (degenerated) plasma (see, e.g., [19]). 2) Short range quantum effects which appears due to the tunneling of electrons and positrons through the
Bohm quantum potential barrier [20]. A study of these and other aspects will be reported elsewhere.

\section{Acknowledgements}

This work has been supported by the Armenian Ministry of Higher Education and Science under Grant No. 87.

\section{References}

[1] A. I. Akhiezer, et al., "Plasma Electrodynamics," Pergamon Press, Oxford, Vol. 1 and 2, 1975,

[2] J. P. Dougherty and O. T. Farley, "A Theory of Incoherent Scattering of Radio Waves by A Plasma," Proceedings of the Royal Society A, Vol. 259, 1960, pp. 79-99.

[3] O. T. Farley, J. P. Dougherty, and D. W. Barron, "A Theory of Incoherent Scattering of Radio Waves by a Plasma. Scattering in A Magnetic Field," Proceedings of the Royal Society A, Vol. 263, 1961, pp. 238-258.

[4] E. E. Salpeter, "Electron Density Fluctuations in a Plasma," Physical Review, Vol. 120, 1960, pp. 1528-1535.

[5] E. E. Salpeter, "Plasma Density Fluctuations in a Magnetic Field," Physical Review, Vol. 122, 1961, pp. 1663-1674.

[6] M. N. Rosenbluth and N. Rostoker, "Scattering of Electromagnetic Waves by a Nonequilibrium Plasma," Physics of Fluids, Vol. 5, 1962, pp. 776-788.

[7] A. G. Sitenko, "Electromagnetic Fluctuations in Plasma," Academic Press, New York, 1967.

[8] A. G. Sitenko and Yu. A. Kirochkin, "Scattering and Transformation of Waves in a Magnetoactive Plasma," Soviet Physics Uspekhi, Vol. 9, 1966, pp. 430-447.

[9] D. E. Evans and J. Katzenstein, "Laser Light Scattering in Laboratory Plasmas," Reports on Progress in Physics, Vol. 32, 1969, pp. 207-271.

[10] J. Sheffield, "Plasma Scattering of Electromagnetic Radiation," Academic Press, New York, 1975.

[11] H. Bindslev, "A Quantitative Study of Scattering from Electromagnetic Fluctuations in Plasmas," Journal of Atmospheric and Terrestrial Physics, Vol. 58, 1996, pp. 983-989.

[12] A. G. Sitenko, "Fluctuations and Wave Scattering in Plasmas (Hydrodynamic and Kinetic Approaches)," Physica B, Vol. 228, 1996, pp. 97-106.

[13] A. G. Sitenko, "Low-Frequency Fluctuations and Electromagnetic Wave Scattering and Conversion in Magnetized Plasma," Proceedings of the Indian National Science Academy A, Vol. 65A, 1999, pp. 481-509.

[14] V. N. Pavlenko and V. G. Panchenko, "Transformation of Electromagnetic Waves in Turbulent Magnetized Plasma," Physica Scripta, Vol. 81, 2010, pp. 065502 (1-4).

[15] U. de Angelis et al., "Scattering and Transformation of Waves in Dusty Plasmas," Physica Scripta, Vol. 98, 2002, pp. 163-167.

[16] L. D. Landau and E. M. Lifshitz, "Electrodynamics of Continuous Media," Pergamon Press, Oxford, 1984. 
[17] N. A. Krall and A. W. Trivelpiece, "Principles of Plasma Physics," McGraw-Hill, New York, 1973.

[18] V. V. Pustovalov and V. P. Silin, "Nonlinear Theory of Waves Interaction in Plasma," (in Russian) Proceedings of the Lebedev Physics Institute of the Academy of Sciences of USSR, Vol. 61, 1972, pp. 42-281.
[19] D. M. Sedrakian and A. A. Avetisyan, "Magnetohydrodynamics of Plasma in the Crust of a Neutron Star," Astrophysics, Vol. 26, 1987, pp. 295-302.

[20] P.K. Shukla and B. Eliasson, "Nonlinear Aspects of Quantum Plasma Physics," Physics-Uspekhi, Vol. 53, 2010, pp. 51-76. 